UDK 78(43)

\author{
Elisabeth Th. Fritz
}

Kommision für Musikforschung der Österreichischen Akademie der Wissenschaften, Wien Komisija za muzikologijo Avstrijske akademije znanosti, Dunaj

\title{
Das Sacrum Imperium Romanum als höfischer Musikraum
}

\section{Sveto rimsko cesarstvo kot dvorni glasbeni prostor}

SUMMARY

For more than thousand years (800-1806) the Holy Roman Empire was the political and culture centre in Europe, dominating not only the space now called middle Europe, but also parts of Italy. Based on a strictly hierarchical feudal system of courts (with the imperial court at the top), which were bound together in a difficult network with changing alliances, the Holy Roman Empire did not change to a "modern" system of state organisation as France or England did at the end of the Middle ages, but exists as feudal based alliance of sovereign states. With the beginning of the $16^{\text {th }}$ century representation as political instrument became more and more important and within courtly arts as poetry, painting and music. Court chapels were founded or reorganized, music became a part of all ceremonies - not only weddings, coronation, but also Reichstage and other imperial events.

For musicians this network of courts but also official meetings, weddings and imperial events were important - for changing to other courts, influences of new style, teaching, playing together with musicians of other court chapels etc.
PovZetek

Več kot tisoč let (800-1806) je bilo Sveto rimsko cesarstvo politično in kulturno središče v Evropi, ki je prevladovalo ne le prostor, ki se danes imenuje Srednja Evropa, temveč tudi dele Italije. Zasnovano na trdnem hierarhičnem fevdalnem sistemu dvorov (s cesarskim dvorom na vrhu), ki so bili povezani v gosto mrežo spreminjajočih se zavezništev, se Sveto rimsko cesarstvo ni spremenilo v "moderen" sistem državnih organizacij, kot sta se Francija ali Anglija ob koncu srednjega veka, temveč je obstajalo kot fevdalno osnovana zveza suverenih držav. Od začetkov 16. stoletja je postala reprezentacija kot politični instrument vedno bolj pomembna, znotraj tega pa pridobivajo na pomenu zlasti poezija, slikarstvo in glasba. Dvorne kapele so se ustanavljale ali reorganizirale, glasba je postala del vseh ceremonij ne le porok, kronanj, temveč tudi parlamentarnih in dvornih dogodkov.

Za glasbenike je bila pomembna ta mreža dvorov in uradnih srečanj, porok in vladarskih dogodkov za izmenjavo med dvori, nove slogovne vplive, poučevanje, skupno muziciranje med glasbeniki različnih dvornih kapel itd.

Wer sich mit der "musikalischen Identität Mitteleuropas" beschäftigt, kann dies nicht, ohne jenes Staatengebilde einzubeziehen, das 1006 Jahre (von 800, der Krönung Karls des Großen, bis 1806, der Aufhebung des Reiches durch Kaiser Franz II.) den identitätsstiftenden Rahmen für diesen Raum, aber auch darüber hinaus, bildete: 
dem Sacrum Imperium Romanum oder Heiligen Römischen Reich (deutscher Nation $^{1}$ ).

\section{Das Reich}

Das Sacrum Imperium Romanum, das "Reich", ist mit heutigem Staats- und Nationenverständnis kaum zu begreifen. Bis zu seinem Ende ein buntes Konglomerat an unterschiedlichen Staatengebilden, die in ebenfalls unterschiedlicher Weise durch ein komplexes Lehens- und Pfründewesen miteinander verbunden waren, entsprach es keineswegs den heutigen Vorstellungen von Staatlichkeit, aber auch nicht dem heute bestehender Staatenbünde wie der Europäischen Union oder den Vereinigten Staaten von Amerika ${ }^{2}$. Getragen durch eine kirchlich legitimierte Reichsidee ${ }^{3}$, basierte es im wesentlichen auf durch einen ritterlich-christlichen Ehrenkodex getragenes Lehensystem und Beziehungsnetzwerk. Der Kaiser, seit der Goldenen Bulle Karls IV. durch die sieben Kurfürsten gewählt, war auf die Loyalität seiner Fürsten ebenso angewiesen, wie auf gute Beziehungen zu den Nachbarländern des Reiches, das aufgrund seiner heterogenen Strukturen wesentlich verletzlicher war als z. B. das seit dem 100jährigen Krieg sich als territorial geeinter Flächenstaat präsentierende Frankreich. Schließlich befanden sich unter den souveränen Fürsten des Reiches nicht wenige, die über die selbe, wenn nicht sogar mehr Macht, Vermögen und Ansehen verfügten als der Kaiser (und nicht selten hatte man in der langen Geschichte des Reiches mit Absicht einen unbedeutenden Fürsten zum Kaiser gewählt, in der Hoffung, die eigene Souveränität in möglichst hohem Maße erhalten zu können ${ }^{4}$ ).

Aus der komplexen Struktur des Reiches folgt einerseits, daß aufgrund der kleinräumigen Binnenstruktur souveräner Staaten von einem grenzenlosen Mitteleuropa keineswegs die Rede sein konnte, und dessen Bewohner und Untertanen ihre "Natio", ihre Herkunft mehrfach definierten mußten. Die Bezeichnung "deutsch" (Heiliges Römisches Reich deutscher Nation) ist demnach keineswegs im einengenden nationalistischen Sinne des 19. und 20. Jahrhunderts zu deuten, sondern als Zugehörigkeit zum Reich und dessen multikultureller und multiethnischer Gemeinschaft, und

\footnotetext{
${ }^{1}$ Die Bezeichnung "deutsch" (Sacrum Imperum Romanum Teutonicum) ist seit dem 15. Jahrhundert in Verwendung und versteht deutsch nicht im national(istisch)en Sinn.

${ }^{2}$ Die meisten Lexika und auch das dtv Wörterbuch zur Geschichte (Konrad Fuchs - Heribert Raabe [Hg.], dtv Wörterbuch zur Geschichte. München ${ }^{6} 1987$, Bd. 2: Stichwort Reich) weichen einer Definition des Heiligen Römischen Reiches aus. Ronald G. Asch ist einer der wenigen, die einen Versuch wagen: "In contrast to France and England, and to a lesser extent also in contrast to the Spanish dominions, the Holy Roman Empire was not a state ruled from a single centre; indeed, it was not really a state at all, but a political 'commonwealth' of an older kind, only loosely bound together and with a multitude of autonomous or semi-autonomous rulers." Ronald G. Asch, Introduction, in: Ronald G. Asch - Adolf M. Birke (ed.), Princes, Patronage, and the Nobility. The Court at the Beginning of the Modern Age c. 1450-1650. London 1991, 29.

${ }^{3}$ Bis in die Frühneuzeit war nicht die Kaiserwahl durch die Kurfürsten, sondern die Krönung durch den Papst in Rom ausschlaggebend für die Legitimation des Amtes. Erst in der Frühneuzeit (v. a. nach Karl V.) war die Wahl ausschlaggebend; in Zeiten konfessioneller Zersplitterung hätte die Legitimation durch ein nur von Teilen der Reichsfürsten anerkanntes geistliches Oberhaupt das lose Staatengebilde des Reiches zusätzlich destabilisiert.

${ }^{4}$ Auch der "kleine" schwäbische Fürst Rudolph von Habsburg war aus diesen Gründen gewählt worden, doch hatten die Kurfürsten nicht mit dem Ehrgeiz Rudolphs und der Durchsetzungskraft seiner Familie gerechnet.
} 
wurde durch eine Herkunftsbezeichnung im engeren Sinn (Tiroler, Sachse etc.) ergänzt. Wenn man bedenkt, daß nicht nur Böhmen, Mähren und Schlesien, sondern bis in das Barock auch große Teile Oberitaliens zum Reich gehörten, wird die Dimension des Staatenbundes deutlich ${ }^{5}$.

Aus der spezifischen Struktur des Reiches resultierte nicht nur seine kulturelle (auch sprachliche) Vielfalt, sondern auch aufgrund der Zusammensetzung aus souveränen Teilstaaten eine plurizentrische Machtstruktur mit zahlreichen Höfen und adeligen Haushaltungen unterschiedlicher Größe, Prachtentfaltung und politischer Gewichtung, die nicht nur durch ein diplomatisches, sondern oft auch familiäres Netzwerk (man denke beispielsweise an die Heiratspolitik der Habsburger) verbunden waren. Im Gegensatz zum französischen Hof, dessen Vorbildstellung unbestritten und der das erstrebenswertes Ziel des Adels war, gab es im Reich mehrere Höfe, die mit dem Kaiserhof in Konkurrenz traten - ein für die kulturelle Entwicklung des Reiches nicht unwesentlicher Faktor.

\section{Hof}

Der Hof war die "familia" ${ }^{6}$ des Herrschers und inkludierte neben den Mitgliedern der Familie (im engeren wie im weiteren Sinn), die Gefolgschaft der Adeligen (Höflinge) wie auch der Bediensteten (Beamte, Diener und Garden) ${ }^{7}$. Er war an die Person des Herrschers gebunden und befand sich immer dort, wo sich dieser aufhielt (nach dem alten Grundsatz "ubi caesar, ibi Roma") ${ }^{8}$. Die starke personelle Bindung brachte es mit sich, daß der Tod des Herrschers auch das Ende seines Hofes bedeutete 9 . Der Hofadel mußte neue Allianzen suchen, und vielen der alten Ratgeber und Vertrauten des verstorbenen Herrschers war angeraten, sich auf ihre Güter zurückzuziehen; die Bediensteten konnten im günstigsten Fall auf eine Übernahme in den Hof des Nachfolgers hoffen, dessen bereits bestehender Hof aufgrund der neuen Würde eine Erweiterung durch in Zeremoniell und Repräsentation erfahrene Kräfte bedurfte. War jedoch keine Kontinuität der Hofhaltung möglich, wurde eine mehr oder minder große Zahl an Bediensteten freigesetzt, die nach Aufgaben an anderen Höfen suchen mußten bzw. (v.a. die Spitzenkräfte in Verwaltung und Kunst) von "Headhuntern" anderer Höfe abengagiert wurden; das enge Netzwerk, das die Höfe

${ }^{5}$ Zur Ausdehnung des Heiligen Römischen Reiches vgl. F. W. Putzger - Egon Lendl - Wilhelm Wagner, Historischer Weltatlas zur allgemeinen und österreichischen Geschichte. Wien 1981.

6 "Familia" im Verständnis der römischen Antike als Hausgemeinschaft bzw. Gemeinschaft aller unter der Verfügungsgewalt des pater familias.

${ }^{7}$ Dazu Jeroen Duindam, The court of the Austrian Habsburgs: locus of a composite heritage, in: Mitteilungen der Residenzen-Kommission der Akademie der Wissenschaften zu Göttingen 8 (1998) 2, $28 f$.

8 " $[. .$.$] In a formal sense, 'the court' was, by definition, wherever the prince happened to take up residence. Once the$ ruler's household had acquired a regular residential base, however, it came to be identified far more colesly with its urban and architectonic settings. Only a fraction of the total number of courtiers was ever actually housed in the ruler's palace. 'The court' included not only the palace buildings, but also the personnel of the household and many of the functions of government, within a single portmanteau meaning." John Adamson, Introduction. The Making of the Ancien-régime Court. 1500-1700, in: John Adamson (ed.), The Princely Courts of Europe. Ritual, Politics and Culture Under the Ancien Régime. 1500-1750. London 1999, 11.

9 Vgl. J. Duindam, siehe Anm. 7, 30. 
des Reiches miteinander verband, begünstigte den raschen Aufbau neuer PatronageKlientel-Beziehungen ${ }^{10}$.

Hatte bereits der Wandel des Fürstenbildes zu Beginn der Frühneuzeit und der dadurch bedingte erhöhte Aufwand für Repräsentation ein Anwachsen des künstlerischen Personals innerhalb der Hofstaate bewirkt, steigerte sich die Größe der Höfe mit dem Wechsel vom herumziehenden Hof bzw. Herrscher zu festen Residenzen deutlich. "Perhaps the most obvious effect of the shift from peripatetic entourage to residential court was its impact on the size of the monarch's household. Even if we confine our analysis to salaried officers, there is a clear trend in almost all of the major European courts towards steady expansion in the two centuries after 1500. The Munich household establishment (Hofstaat) of the Wittelbach Dukes of Bavaria, for example, burgeoned from a mere 162 in 1508 to 866 in 1571. By the late seventeenth century, it stood at well over a thousand, making it the second largest in the Holy Roman Empire and one of the largest in Europe. The Medici ducal household at Florence rose from some 168 in 1564 to a peak of 792 in 1695, before reducing in scale in the early eighteenth century. [...]" ${ }^{11}$ Ähnlich war die Entwicklung des Kaiserhofes: Unter Maximilian I. ca. 500 Personen umfassend, sank die Zahl unter Ferdinand I. leicht ab, um unter Maximilan II. wieder einen Stand von ca. 500 Personen zu erreichen. Bis zur Regierungszeit Leopolds I. war dann der Hofstab mit ca. 600 Personen in seiner Größe relativ stabil. Erst in Folge der strukturellen (Neu-)Entwicklung des Hofes unter Leopold I. und in der Regierungszeit seiner beiden Söhne stieg die Zahl auf 2000 bis 2500 bis zur Mitte des 18. Jahrhunderts $a^{12}{ }^{12}$. Diese Steigerung ist jedoch weniger auf den Zuzug von Adel an den Hof (wie z. B. in Frankreich unter Ludwig XIV.) zurückzuführen, als vielmehr auf den Ausbau von Verwaltungspersonal, Dienerschaft und nicht zuletzt künstlerischem bzw. wissenschaftlichem Personal (Theater und Bibliotheken hatten für ihre Entwicklung schließlich eine feste Residenz zur Voraussetzung).

Im Gegensatz zum französischen Hof (und somit auch im Gegensatz zu den betreffend das Reich zunehmend kritisch ${ }^{13}$ betrachteten Theorien von Norbert Elias ${ }^{14}$ )

${ }^{10}$ Bei Bewerbungen an den Wiener Hof wurde z. B. immer wieder auf bereits bestehende Beziehungen zum Hof (die Nennung eines Gewährsmannes wie Hinweise auf Patronagemakler) hingewiesen, wie auf Erfahrung in Diensten bei anderen Höfen (vgl. Obersthofmeisteramtsprotokolle bzw. Obersthofmeisteramtsakten im Haus-, Hof- und Staatsarchiv in Wien).

${ }^{11}$ John Adamson, siehe Anm. 8, 12.

${ }_{12}$ J. Duindam, siehe Anm. 7, 30f.

${ }^{13}$ Zur Kritik an Norbert Elias vgl. u.a. Ronald G. Asch, Introduction, in: Ronald G. Asch - Adolf M. Birke (ed.), Princes, Patronage, and the Nobility. The Court at the Beginning of the Modern Age. c. 1450-1650. Oxford-New York 1991, S. 1-38. S. 2-3: "Nevertheless, we still know little about the court as a political and social institution. What we do know is to a large extent due to the work of the historian and sociologist Norbert Elias - one could almost say he was the first to discover the court as an area of research. Elias sees the court essentially as an instrument which served to 'domesticate' the nobility and to consolidate the absolutist state. His interpretation is based largely on the court society at Versailles during the reign of Louis XIV and the contemporary analysis of it provided by the duc de Saint-Simon. [...] In the final analysis the model Elias puts forword rests too heavily on the assumption of a fundamental opposition between ruler and nobility, an opposition that made it advisable for the king or prince to reduce the local influence of the nobility by drawing them to the court."

${ }^{14}$ Norbert Elias, Die höfische Gesellschaft. Untersuchungen zur Soziologie des Königtums und der höfischen Aristokratie. Frankfurt am Main 1983 (Surkamp-TB Wissenschaft 423). 
strebte der Reichadel nur eingeschränkt an den Kaiserhof, sondern imitierte diesen (bzw. - je nach aktueller politischer Ausrichtung - den französischen Hof) in der eigenen Residenz. Dadurch ergab sich für den Bereich der Hofkünste (Dichtung, Malerei, Architektur und Musik) die Möglichkeit der Multiplikation des Arbeitsgebietes. Ein kodifizierter höfischer Stil ermöglichte den Künstlern mit diesem erlernten Vokabular an allen Höfen des Reiches tätig zu $\operatorname{sein}^{15}$. Von einem verbindlichen Reichs- oder Kaiserstil ${ }^{16}$ zu sprechen, ist jedoch problematisch, vielmehr handelte es sich um einen höfischen Stil, dessen Vokabular je nach Ausrichtung des Hofes (nach Italien, nach Wien oder nach Frankreich) modifiziert wurde.

\section{Musik und Musiker}

Ein mehr oder minder festes Engagement an einer Kirche oder einem Hof zu bekommen, war seit jeher für einen Musiker ein erstrebenswertes Ziel, da es ihm nicht nur soziale Sicherheit, sondern auch Ansehen und die Möglichkeit des sozialen Aufstieges bot (nicht selten wurden Musiker in Hofdiensten in den Adelsstand erhoben). Im Gegensatz zum herumziehen Musiker, dem Vaganten, dem die schützende Hand eines "Patrons" (eines Grundherrn, Adeligen, Regenten etc.) fehlte und der somit auch bar grundlegender bürgerlicher Rechte war, konnte der in höfischen Diensten stehende Musiker darauf zählen, daß das Ansehen seines Dienstherren auch ein wenig auf ihn "abfärbte". Unter diesen Auspizien wird die Beliebtheit von Titularund Ehrenstellen, durch die ein Patronage-Verhältnis suggeriert wurde, das zwar de facto nicht bestand, dem Besitzer des Titels jedoch an anderen Höfen bzw. Institutionen Tür und Tor öffnete ${ }^{17}$, verständlich. Reisenden Virtuosen, die sich nicht der schützenden Hand eines Patrons versichern konnten, blieb jedoch bis zum Endes des ancien régime der schlechte Ruf der Vaganten anhaften: Personen die man nicht in die Höfe hineinlassen wollte und denen daher auch der Musikraum des Höfenetzwerkes weitgehend versperrt blieb. Auch die Mozarts, obwohl aus einem Hof kommend, sich jedoch als reisende Virtuosen primär präsentierend, blieben davor nicht ver-

${ }^{15}$ Vgl. dazu Elisabeth Th. Hilscher, „...dedicata alla sacra cesarea maestà ..... Joseph I (1678-1711) und Karl VI. (16851740) als Widmungsträger musikalischer Werke - zum historischen und geistesgeschichtlichen Umfeld der Widmungskompositionen, in: Studien zur Musikwissenschaft 41 (1992) 96-101; bzw. Elisabeth Th. Hilscher, Mit Leier und Schwert. Die Habsburger und die Musik. Graz-Wien-Köln 2000, v. a. 44-52 und 89-97.

${ }^{16}$ Diese These stammt vom Kunsthistoriker Hans Sedlmayr (Die politische Bedeutung des deutschen Barocks. Der "Reichsstil", in: Gesamtdeutsche Vergangenheit. Festgabe für Heinrich Ritter von Srbik. München 1938, 126-40) und wurde von der Musikwissenschaft aufgegriffen (vgl. Friedrich W. Riedel, Der "Reichsstil" in der deutschen Musikgeschichte des 18. Jahrhunderts, in: Bericht über den internationalen musikwissenschaftlichen Kongreß Kassel 1962. Kassel 1963, 34-36). Von der Kunstgeschichte mittlerweile als nicht haltbar verworfen, kann sich die Musikwissenschaft jedoch immer noch nicht von dieser These eines durch den Kaiserhof in Wien und seiner Hofmusikkapelle geprägten und von allen (resp. vielen) Hofkapellen im Reich imitierten Stiles trennen. Vgl. dazu auch den Artikel Kaiserstil, in: Oesterreichisches Musiklexikon 2 (2003) 935f.

${ }^{17}$ Neben Kammervirtuosen-Titel, Kammer-Kompositor, Kammer-Kapellmeister etc. waren auch Widmungen sehr beliebt; von Antonio Lotti, der Joseph I. Duetti, Terzetti, e Madrigali à piu voci [...], Venedig 1705, widmete, wird berichtet, er habe die goldene Kette mit dem Bildnis des Kaisers, die er als Dank für die Dedikation erhalten hatte, stolz bei jedem offiziellen Anlaß getragen, v. a. aber jährlich an jenem Tag, "an welchem er dem k.k. Gesandten in Venedig seinen Ehrenbesuch abzulegen pflegte." Anton Schmid, Der berühmte Tonsetzer Antonio Lotti, in: Österreichische Blätter für Literatur und Kunst (Hg. A. Schmid) 2 (1845) 587, zitiert bei Elisabeth Th. Hilscher, "... dedicata ...", siehe Anm. 15, 123. 
MUZIKOLOŠKI ZBORNIK • MUSICOLOGICAL ANNUAL XL

schont. Als Wolfgang Amadeus 1771 nach seinem Triumph mit Ascanio in Alba in Mailand hoffte, von Erzherzog Ferdinand in dessen Hof aufgenommen zu werden, riet Maria Theresia ihrem Sohn vom einem Engagement des unsteten, sich beständig auf Reisen befindlichen Komponisten $a b^{18}$. Hofmusiker waren Dienstleister in Sachen Repräsentation und ihre stete Anwesenheit und Verfügbarkeit für den Herrscher notwendig.

Dennoch konnten die Hofmusiker (und v. a. die Spitzenkräfte unter ihnen) keineswegs auf eine ruhiges Leben an einem Ort hoffen. Schon seit dem Spätmittelalter, als Repräsentation und Zeremoniell immer wichtiger zur Präsentation von Macht und Ansehen wurden, hatten Musiker ihre Dienstherren zu allen großen Ereignissen im Reich zu begleiten. Da bis zur Regierungszeit Kaiser Ferdinands II. das Heilige Römische Reich ein Wanderkönigtum war, d.h. nicht nur der Herrscher zwischen mehreren Residenzen hin- und herreiste, sondern auch die Reichstage an unterschiedlichen Orten stattfanden, mußten nicht nur die Musiker in kaiserlichen Diensten quer durch das Reich fahren; Paul Hofhaimer klagte rückblickend, er hätte mit Maximilian "wie ein Zigeuner durchs Land ziehen müssen " ${ }^{19}$. Walter Senn stellte anläßlich der MaximilianAusstellung in Innsbruck 1969 ein Itinerar der Hofmusikkapelle zusammen, das anschaulich die intensive Reisetätigkeit des maximilianeischen Hofes vor Augen führt ${ }^{20}$ :

1495 Worms, Reichstag (nur Hofhaimer und die Instrumentalisten?)

1496 Augsburg, ein Teil der Kapelle übersiedelt nach Wien

1498: Freiburg, Reichstag, an dem auch die Kantorei Friedrich des Weisen anwesend ist

1500 Augsburg (März-August), Reichstag; dann Tirol, Bruneck (30.November)

1501 Linz (Januar-März), Nürnberg (Reichstag), Innsbruck

1503 Innsbruck (September, Oktober), Augsburg (Dezember?)

1504 Augsburg (nur Instrumentalisten?)

1505 Hagenau im Elsaß, Köln (Reichstag)

1506 Augsburg (nur Instrumentalisten?)

1507 Konstanz, Reichstag

1510 Augsburg, Reichstag

1511 Innsbruck (Herbst bis Dezember)

1512 Worms, Koblenz, Trier (Reichstag), Augsburg, Köln (Reichstag)

1515 Wien (Fürstenkongreß)

1516 Wien, Augsburg

1518 Innsbruck, Augsburg (Reichstag), Innsbruck

Dieses Itinerar betraf nicht nur die Mitglieder des kaiserlichen Hofes, sondern auch alle Reichsfürsten und deren Höfe, handelte es sich bei den meisten Stationen doch um offizielle Termine des Reiches wie Reichstage oder Fürstenkongreß, Hochzeiten, Begräbnisse. Für die Musiker bedeuteten diese Reisen (abgesehen von den

\footnotetext{
${ }^{18}$ Dazu: E. Th. Hilscher, Mit Leier, siehe Anm. 15, 174.

${ }^{19}$ Gernot Gruber, Beginn der Neuzeit, in: Rudolf Flotzinger - Gernot Gruber (Hg.), Musikgeschichte Österreichs 1. WienKöln-Weimar 1995, 188.

${ }^{20}$ Walter Senn, Maximilian und die Musik, in: Ausstellung Maximilian I. Innsbruck 1969
} 
Strapazen und Kosten) ein Zusammentreffen mit den besten Musikern der Zeit, das nicht selten (auch unter dem Einfluß der Dienstherren) in einem Um-die-Wette-Musikzieren endete. Neben der Überprüfung des eigenen Könnens brachte es (v.a. für Musiker an kleineren Höfen) eine Erweiterung des musikalischen Horizontes und die Möglichkeit, Kontakte zu knüpfen: nicht nur, um an größere Höfe zu wechseln, sondern auch, um bei bedeutenden Musikerpersönlichkeiten die eigenen Fähigkeiten perfektionieren zu können. Nicht selten stürzten sich die Herrscher in Schulden, nur um bei diesen offiziellen Anlässen ihrem Stand und ihrer Würde entsprechend repräsentieren zu können. Schon Maximilian I., einer der ersten Fürsten, die Kultur, und v. a. Musik, als Mittel der Propaganda einsetzten, verwendete seine Hofmusiker anläßlich der Wahl seines Sohnes Philipp zum römischen König 1485/86 in Aachen bzw. Frankfurt am Main, um sich als erster Fürst des Reiches zu präsentieren ${ }^{21}$. Meist wurde die Musik zu Krönungsfeierlichkeiten nicht ausschließlich durch die kaiserliche Kapelle bestritten; ortsansässige Musiker (z. B. der Hauptkirchen) und Hofkapellen befreundeter Reichsfürsten ergänzten die kaiserliche Kapelle. Da Bankette, Bälle, Serenaden etc. in den zu diesem Zweck eilig in den jeweiligen Reichsstädten errichteten Residenzen der bedeutenden Reichsfürsten große Feierlichkeiten des Reiches begleiteten, war die Möglichkeit des künstlerischen Austausches sehr groß ${ }^{22}$.

Große Bedeutung im kulturellen Transfer ist dem Austausch bzw. Teil-Austausch von Hofstaaten z. B. im Zuge von Hochzeiten zu geben. Die Prinzessinnen kamen nicht nur mit einem kleinen eigenen Hofstaat ausgestattet an die jeweiligen Höfe, sondern traten in der Folge meist als Mäzeninnen (Patronagemakler) ihrer ehemaligen Landsleute an den Höfen des Reiches auf. Als Beispiele sollen hier nur die bayerischen Prinzessinnen (Maria und Maria Anna) genannte werden, die im 16. Jahrhundert in die innerösterreichische Linie des Hauses Habsburg einheirateten bzw. die Medici- und Gonzaga-Prinzessinnen (allen voran die beiden Eleonoren ${ }^{23}$ ), die durch ihren aktiven Einsatz in kulturellen und v. a. musikalischen Angelegenheiten einen wesentlichen Anteil an der Entwicklung des musikalischen Barock in Österreich hatten. Dieser Transfer funktionierte ebenfalls in umgekehrter Richtung, also aus dem Reich heraus z. B. an italienische Höfe außerhalb des Reiches oder nach Polen.

\footnotetext{
${ }^{21}$ Gernot Gruber, siehe Anm. 19, 184.

22 "Mit seinem früh verstorbenen Sohn [Philipp dem Schönen] war Maximilian noch zweimal zusammengetroffen. Beide Treffen wurden natürlich auch zu musikalischen Festen, deren in Chroniken entsprechend gedacht wurde: 1503 weilte Philipp mit seinem Gefolge, darunter 39 Kapellisten und Hofmusiker, auf der Rückreise aus Spanien in Hall in Tirol. Wie bei derartigen Anlässen üblich, wirkten die Kapellen beider Herrscher gemeinsam an der Ausgestaltung höfischer Festlichkeiten. Einen solchen Anlaß bot das Totenamt für Maximilians Schwager, Hermes von Mailand, in Innsbruck. [...] Das letzte Treffen zwischen Maximilian und Philipp fand dann anläßlich des Abschlusses des Vertrages von Blois mit dem französischen König in Hagenau im Elsaß statt. Wiederum musizierten die anwesenden Kapellen gemeinsam oder abwechselnd bei offiziellen Festlichkeiten. Ähnliches ereignete sich 1512 am Reichstag in Trier, als die Kapellen Maximilians und Herzog Ulrichs von Württemberg am Palmsonntag Offizium und Passion figurierten." Gernot Gruber, siehe Anm. 19, 191.

${ }^{23}$ Eleonora Gonzaga (I), die zweite Frau Kaiser Ferdinands II., und Eleonora Gonzaga (II.), die dritte Frau Kaiser Ferdinands III., werden mit der Einführung von Oper und Oratorium in den österreichischen Ländern in Verbindung gebracht; Eleonora (II.) verfügte in ihrer langen Witwenschaft über eine hervorragende Hofmusikkapelle, die durchaus in Konkurrenz mit kaiserlichen ihres Stiefsohnes Leopold I. stand.
} 
MUZIKOLOŠKI ZBORNIK - MUSICOLOGICAL ANNUAL XL

Einen Sonderfall stellt die Anstellung Heinrich Isaacs durch Maximilian I. dar: Nach der Vertreibung der Medici aus Florenz ohne Herrschaft, sicherte sich der Kaiser den prominenten Musiker durch eine (damals singuläre) Anstellung als Hof-Komponist, ließ im jedoch jegliche Reisefreiheit, da der Kaiser erkannte, daß Isaac für Ruf und Ansehen des Kaisers so nützlicher sein konnte, als durch eine feste räumliche Bindung an den $\mathrm{Hof}^{24}$.

Studienreisen von Musikern, oft aufgrund eines Stipendiums, das ein adeliger Mäzen gestiftet hatte, oder im Auftrag des Dienstherren dienten ebenfalls dem Kulturtransfer, waren sie doch nicht selten mit dem Auftrag, die neuesten Musikalien für die Kapelle "zu Hause" (d.h. im Reich) anzukaufen bzw. sich um neues künstlerisches Personal umzusehen. In jedem Fall war die Mobilität der Musiker jedoch an die Zustimmung bzw. den ausdrücklichen Wunsch eines Dienstherren oder Mäzens, eines Patrons, gebunden, dessen Sendschreiben nicht nur die zahlreichen Grenzschranken, sondern auch die Türen zu einflußreichen Kreisen öffneten. Als adeliges Pendant zu den Studienreisen der Musiker ist die Cavalierstour zu erwähnen, seit der Frühneuzeit von vielen deutschen Fürsten gepflegt, die meist nach Italien führte und ebenfalls deutlich kulturell (Architektur, Malerei, Literatur und Musik) ausgerichtet war. Nicht selten knüpften junge Adelige Kontakte zu Künstler, die sie später an ihre Höfe engagierten ${ }^{25}$.

Wer als Musiker das Glück hatte, in ein höfische Patronage-Klientel-Verhältnis zu kommen, verließ dieses selten freiwillig, sondern versuchte Karriere durch "courthopping", das Wechseln zu immer bedeutenderen Höfen des Reiches, zu machen ${ }^{26}$. Kontaktnahmen bei offiziellen Anlässen des Reiches, die seit dem Spätmittelalter ohne Mitwirkung der Hofkapellen undenkbar waren, erleichterten unter Nutzung des höfischen Netzwerkes des Reiches einen Wechsel zu anderen Dienstherren. Ein durch eine kodifizierte Amtsethik vorgegebenes Vokabular, das für alle Hofkünste den Rahmen vorgab, wirkte als verbindliche Klammer. So ergab sich für Musiker innerhalb der Höfe des Heiligen Römischen Reiches ein großer Musikraum, der, wenn man sich der Patronage eines Fürsten versichern konnte, beeindruckende Curricula ermöglichte.

\footnotetext{
${ }^{24}$ Vgl. dazu Isaac, Heinrich, in: Oesterreichisches Musiklexikon 2 (2003) 867f.

${ }^{25}$ Vgl. dazu Theophil Antoniceks Angaben zur Cavalierstour des späteren Kaisers Ferdinand II. (Italienische Musikerlebnisse Ferdinands II. 1598, in: Anzeiger der phil.-hist. Klasse der Österreichischen Akademie der Wissenschaften 1967. Graz-Wien-Köln 1968, 91-111 (= Mitteilungen der Kommission für Musikforschung 18).

${ }^{26}$ Riskantes "Court-hopping" bzw. zu hohes Pokern bei Anstellungsverhandlungen konnte jedoch auch zu beträchtlichen Karriererückschlägen führen; ein prominentes Beispiel dafür bietet die Karriere von Orlando di Lasso (für diesen Hinweis danke ich Bernhold Schmid).
} 